Research Article

www.jestr.org

\title{
Environmental Indexes and Financial Ratios
}

\author{
S. Savvidis* and D. Ginoglou \\ University of Macedonia, Dep. of Accounting and Financ, 156 N. Egnatia Str., 54006 Thessaloniki,Greece
}

\begin{abstract}
To date, accounting and financial sciences have attempted to assess companies using financial ratios and other techniques that evaluate only the front office, without considering its impact to the environment. Recently, this assessment has been expanded to include new concepts, known as corporate social responsibility and social balance. These new concepts are usually viewed and studied using a multidisciplinary perspective with an aim to update the current and future value of the company. In this evolving scientific field a considerable effort has been made to objectively record and calculate the environmental impact of a company's activities by integrating these elements into a new form of economic balance sheet. The present paper seeks to examine the presence of weighted environmental indexes and explores the process by which they can be used to evaluate a company.
\end{abstract}

Keywords: Econophysics, Enviromental indexes, financial..

\section{Introduction}

Until the last half of the $20^{\text {th }}$ century, an organization's main goal was to ensure its profit and financial state without considering the impact of its relevant applications and/or productions to the environment. In fact, the single concern was how to find available and cheap natural resources, to extract them and use them efficiently. Few measures were taken to ensure that a company was environmentally friendly, not even measuring the footprint of the process done.

It was in the middle of the $20^{\text {th }}$ century when it became evident that the extreme consumption of the environments resources, leads to irreversible environmental disruption.

The reason for this disruption is the accumulation of environmental misuse from previous generations, as well as, current neglect. Organizations started to realize that it is for their benefit to protect the environment, and at least, be sure that the rate of environmental consumption is smaller or even equal to the rate the environment restores itself.

The new term born was "sustainable development" and firstly talked about that the Prime Minister of Norway, Gro Harlem Brundtland (1). The main issue now is how to satisfy present needs without risking or undermining next generations' capability of satisfying their needs. The solution to this problem demands new way of thinking, innovative choices and use of brand new technology.

\subsection{Our Idea}

At that point, the following idea came up: currently, all we can tell about an organization is what we know from its

\footnotetext{
*E-mail address: s.savvidis@avakas.com

ISSN: 1791-2377 @ 2011 Kavala Institute of Technology. All rights reserved.
}

financial statements and/or other financial data, expressed in global standards forms. Regarding the environmental behavior, we have a blur picture since at the moment there are no obligatory regulations an organization has to follow. Still, some do keep up with some environmental standards, like GRI for instance.

We all sense that financial and environmental results have a strong interconnection, since if we had no action neither we would have financial transactions, nor environmental footprint. So, is it possible to find a model that connects them? We also sense that such a model if it exists, it will not be something simple, linear, but instead non linear and hard to define its parameters. Having all these in mind we decided to search a possible connection by using neural networks.

\subsection{Financial Ratios}

Financial statement analysis is important to boards, managers, payers, lenders, and others who make judgments about the financial health of organizations. One widely accepted method of assessing financial statements is ratio analysis, which uses data from the balance sheet and income statement to produce values that easily interpret financial situation (2).

In fact, most organizations routinely evaluate their financial condition by calculating various ratios and comparing the results to those for previous periods, looking for differences that could indicate a meaningful change in financial condition. Another usage is to compare their current ratios with those so called as "market leaders", in order to examine if they are on the right developmental path.

There are different financial indicators measure different dimensions of financial performance, such as profitability and liquidity, and all of this information is needed to make 
an informed judgment about the financial health of an organization (3).

To select financial performance dimensions, five topselling textbooks on financial management were reviewed $(2,3,4,5,6)$. Not surprisingly, there was substantial overlap and it was relatively straightforward to establish four preliminary dimensions of financial performance: liquidity, capital structure, activity and profitability.

In our case we selected to examine:

Liquidity with

a) Current ratio $=\frac{\text { CurrentAssets }}{\text { CurrentLiabilities }}$,
b) Quick ratio $=\frac{\text { CurrentAssets }- \text { Inventory }}{\text { CurrentLiabilities }}$

Capital Structure with

a) Total Debt $/$ Total Assets $=\frac{\text { TotalLiabilities }}{\text { TotalAssets }}$

b) Equity Financing $=\frac{\text { FundBalance }}{\text { TotalAssets }}$

c) Cash Flow to Total Debt = NetIncome + DepritiationExpences TotalLiabilities

Activity with

a) Total Asset Turnover $=\frac{\text { TotalOperating Revenue }}{\text { TotalAssets }}$

b) Fixed Asset Turnover $=\frac{\text { TotalOperationg Re venue }}{\text { NetFixedAs sets }}$

c) Current Asset Turnover $=\frac{\text { TotalOperating Re venue }}{\text { CurrentAssets }}$

and Profitability with

a) Operating Margin

TotalOperating Revenue-OperatingExpences TotalOperating Revenue

b) Return on Assets $=\frac{\text { NetIncome }}{\text { TotalAssets }}$

\subsection{Environmental Performance Indicators - EPI (Sustainability Indicators, Green Indexes)}

Environmental Performance Indicators (EPI) have the potential to turn the generic concept of sustainability into action. Though there are disagreements among those from different disciplines, these disciplines and international organizations have each offered measures or indicators of how to measure the concept.

Various ways of operationalizing or measuring sustainability have been developed. During the last 10 years there has been an expansion of interest in EPI systems, both in industrialized and, albeit to a lesser extent, in developing countries. EPIs are seen as useful in a wide range of actors: international and intergovernmental bodies; national governments and government departments; economic sectors; administrators of geographic or ecological regions; communities; nongovernmental organizations; and the private sector.
EPI processes are supported and driven by the increasing need for improved quality and regularly produced information with better spatial and temporal resolution.

At the heart of the debate over different indicators are not only different disciplinary approaches but also different views of development. Some indicators reflect the ideology of globalization and urbanization that seek to define and measure progress on whether different countries or cultures agree to accept industrial technologies in their eco-systems. Other approaches, like those that start from international treaties on cultural rights of indigenous peoples to maintain traditional cultures, measure the ability of those cultures to maintain their traditions within their eco-systems at whatever level of productivity they choose.

Currently, main agencies around the world dealing with EPIs are United Nations Environmental Protection Agency (UN EPA), European Environment Agency (EEA), Environmental Permitting Regulations (EPR), Eco Management and Audit Scheme (EMAS) and other organizations.

Although no global standard is yet in action, as mentioned previously, there are standards brought up by individual organizations and companies that are able to collect, classify, analyze and report EPIs. The main two differences amongst them are the extension of information handled and the area of main interest given. For example, standard IPIECA (International Petroleum Industry Environmental Conservation Association) is greatly interested for the consequences of oil and pays less attention for other forms of energy consumption, e.g. electricity or gas.

Standards that are key players at the moment globally are:

a) AccountAbility, with three standards in action

AA1000APS (2008) AcountAbility Principles Standard

AA1000AS (2008) Assurance Standard and

AA1000SES (2005) Stakeholder Engagement Standart

b) IPIECA (International Petroleum Industry Environmental Conservation Association)

c) DEFRA (Department for Environment, Food and Rural Affairs - Govern. Depart. in UK )

d) FTSE4GOOD (Financial Times and the London Stock Exchange)

e) Deloitte and Touche Tohmatsu

f) ISO26000 (International Organization for Standardization)

g) ACCA (Association of Chartered Certified Accountants) and finally

h) GRI (Global Reporting Initiative)

From the above extended list, we chose to use in our research the GRI standard, since most organizations in Greece submit reports using it. From the list of all the EPI the standard has, we picked up two, the first dealing with energy and the second with water management:

a) Aspect Energy

EN3 Direct energy consumption by primary energy source

b) Aspect Water

EN8 Total water withdrawal by source

\section{Proposed Method}

An Artificial Neural Network (ANN) is a system based on the operation of biological neural networks, in other words, is an emulation of biological neural system. Artificial Neural 
Networks have different architectures, which consequently lead to different types of algorithms and problem solving, and are among the newest technologies in the finance's statistics toolbox. The field is highly interdisciplinary, but our approach will restrict the view to the financing perspective, in which ANN serve two important functions: a) pattern classifiers and $b$ ) nonlinear adaptive filters.

An ANN is an adaptive, nonlinear system that learns to perform a function (an input/output map) from data. Adaptive means that the system parameters are changed during operation, normally called the training phase. After the training phase the ANN parameters are fixed and the system is deployed to solve the problem at hand (the testing phase). The ANN is built with a systematic step-by-step procedure to follow some implicit internal constraint, which is commonly referred to as the learning rule. The input/output training data are fundamental in neural network technology, because they convey the necessary information to "discover" the optimal operating point. The nonlinear nature of the neural network processing elements provides the system with lots of flexibility to achieve practically any input/output function.

The performance of an ANN hinges heavily on the data. If data do not cover a significant portion of the operating conditions or if they are noisy, then ANN technology is probably not the right solution. On the other hand, if there is plenty of data and the problem is poorly understood to derive an approximate model, then neural network technology is a good choice.

\subsection{The Biological Model}

ANN emerged after the introduction of simplified neurons by McCulloch and Pitts in 1943 (7). These neurons were presented as models of biological neurons and as conceptual components for circuits that could perform computational tasks. The basic model of the neuron is founded upon the functionality of a biological neuron (figure 1a).
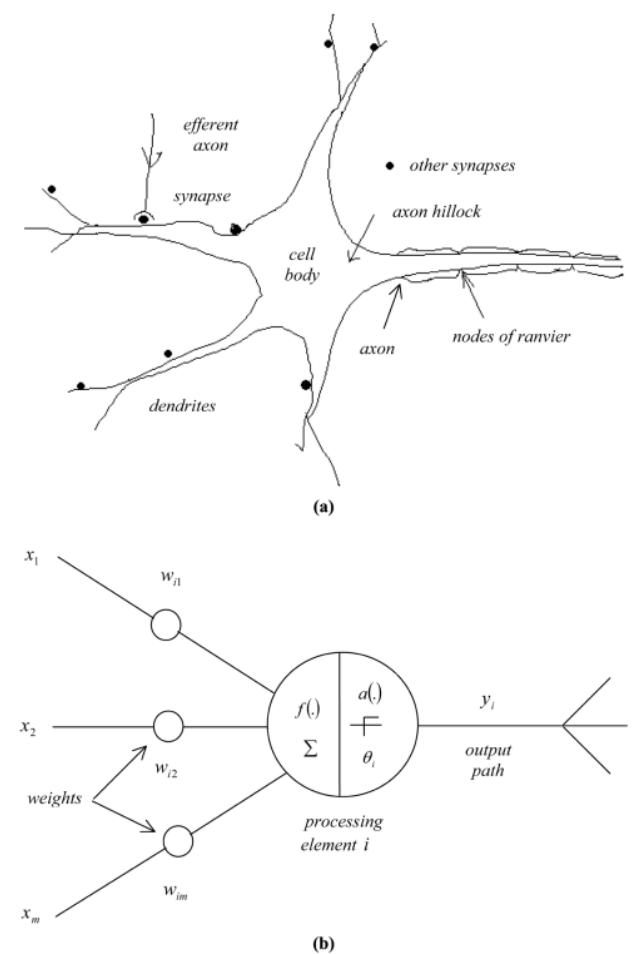

Fig. 1. Biological and Mathematical Neuron

\subsection{The Mathematical Model}

The functional model of the biological neuron has three basic components:

a) Weights,

b) A linear combination and

c) An activation function.

An acceptable range of output is usually between 0 and 1 , or -1 and 1 and is calculated as the output of the neuron from the activation function. Mathematically, this process is described in the figure $1 \mathrm{~b}$, where the output of the neuron, $y_{i}$, would therefore be the outcome of activation function $\alpha$ (.). In our research we chose to use the sigmoid function since it seems it works well enough in our area of interest.

Supervised learning results in an adjustment of the weights of the connections between units, according to some modification rule, for example:

$$
\Delta w_{j k}=\gamma y_{j}\left(d_{k}-y_{k}\right),
$$

where $\gamma$ is a positive constant of proportionality, representing the learning rate, $d_{k}$ is the desired activation provided by data (Widrow-Hoff rule or Delta rule). Since we are using nonlinear activation functions, we use a generalised delta rule. The error measure $E_{p}$ is defined as the total quadratic error for pattern $\mathrm{p}$ at the output units.

\subsection{Back-propagation}

The application of the generalised delta rule involves two phases: During the first phase the input $\mathrm{x}$ is presented and propagated forward through the network to compute the output values $\mathrm{y}_{\mathrm{po}}$ for each output unit. This output is compared with its desired value $d_{o}$, resulting in an error signal $\delta_{\mathrm{po}}$ for each output unit. The second phase involves a backward pass through the network during which the error signal is passed to each unit in the network and appropriate weight changes are calculated.

Backpropagation can be applied to networks with any number of layers, just as for networks with binary units it has been shown $(8,9,10)$ that only one layer of hidden units success to approximate any function with finitely many discontinuities to arbitrary precision, provided the activation functions of the hidden units are non-linear (the universal approximation theorem). In most applications a feed-forward network with a single layer of hidden units is used with a sigmoid activation function for the units.

\subsection{Learning rate and momentum}

For practical purposes we chose a learning rate that is as large as possible without leading to oscillation. One way to avoid oscillation at large, is to make the change in weight dependent of the past weight change by adding a momentum term $(11,12)$.

\section{Discussion}

In order to try our model, we chose to use a:

a) Multi Layer Perceptron (MLP) model with one hidden layer and six neurons

b) Sigmoid activation function

c) Back Propagation training algorithm 
as seen in figure 2 .

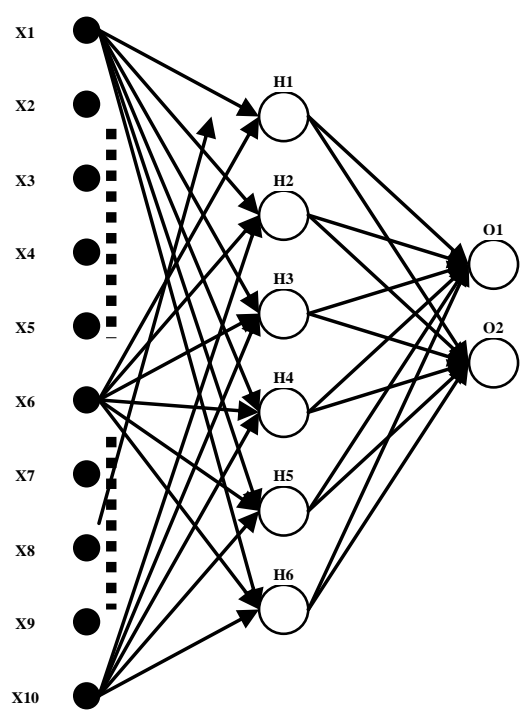

Fig. 2. Out test model

If we find a realistic model that verifies out data, then we will be able to act in the reverse scheme, like an if-then-else scenario at a spreadsheet. It will allow us to decide for environmental critical projects and their extension before they are build up, explore possible limits of existing ones and in general unify the information we can have for an organization.

If on the other hand the chosen model can not verify our data, we will evaluate the error vector and decide out next step. If the error seems to be manageable, we will try different number of neurons in the hidden layer and/or more exotic training algorithm.

If finally the error seems to be unmanageable, we will change our direction to use other statistical tools, like for example data envelopment analysis (DEA).

\section{References}

1. Agassi, J. (1991). The Gro Brundtland Report (1987) Or, The Logic of Awesome Decisions. International Review of Sociology, Monographic On Modernization Theory: Monographic Series, 3 Rome: Borla, 213-226.

2. Garrison, Ray H., Noreen, E., Brewer P. C. (2009). Managerial Accounting. UK: McGrawHill.

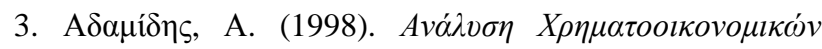

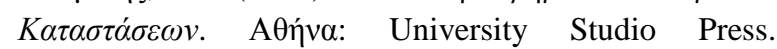
Adamidis, A. (1998). Analysis of financial situations. Athens: University Studio Press.

4. Horngren, C.T. \&Olivar, S. (2009). Managerial Accounting. UK: Prentice Hall.

5. Kaplan, R.,S Atkinson, A., Young, S. (2003). Management Accounting. UK: Prentice Hall.

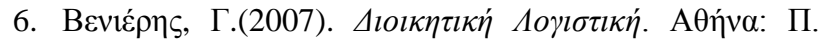
I $\omega \alpha v v i ́ \delta$ ov \& $\Sigma 1 \alpha$ O.E

7. McCulloch, W. S. and Pitts, W. H. (1943). A logical calculus of the ideas immanent in nervous activity.
Bulletin of Mathematical Biophysics, 5,115-133.

8. Hornik, K Stinchcombe M \& White, H. (1989). Multilayer Feedforward Networks are Universal Approximators. Neural Networks, 2, 359-366.

9. Funahashi, K. (1989). On the approximate realization of continuous mappings by neural networks. Neural Networks, 2, 183-192.

10. Hartmann, E.J., Keeler, J.D., Kowalski, J.M. Layered (1990). Neural networks with gaussian hidden units as universal approximators. Neural Computation, 2 210215.

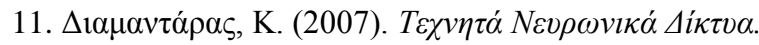

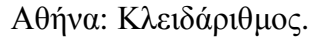
Diamantaras, K. (2007). Artificial neural networks. Athens: Klidarithmos.

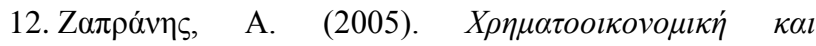

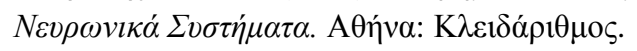

Zapranis, A. (2005). Financial and neural systems. Athens: Klidarithmos. 